Internat. J. Math. \& Math. Sci.

Vol. 24, No. 7 (2000) 469-479

0161171200004087

(C) Hindawi Publishing Corp.

\title{
ORDERED GROUPS WITH GREATEST COMMON DIVISORS THEORY
}

\author{
JIŘí MOČKOŘ
}

(Received 15 October 1999)

\begin{abstract}
An embedding (called a GCD theory) of partly ordered abelian group $G$ into abelian l-group $\Gamma$ is investigated such that any element of $\Gamma$ is an infimum of a subset (possible non-finite) from $G$. It is proved that a GCD theory need not be unique. A complete $G C D$ theory is introduced and it is proved that $G$ admits a complete GCD theory if and only if it admits a GCD theory $G \longrightarrow \Gamma$ such that $\Gamma$ is an Archimedean l-group. Finally, it is proved that a complete GCD theory is unique (up to $o$-isomorphisms) and that a po-group admits the complete GCD theory if and only if any $v$-ideal is $v$-invertible.
\end{abstract}

Keywords and phrases. po-groups, l-groups, GCD-theory, complete GCD-theory.

2000 Mathematics Subject Classification. Primary 13F05, 06F15.

1. Introduction. The problem of embedding of partly ordered abelian directed group (po-group, shortly) $G$ into an abelian lattice ordered group (l-group) $\Gamma$ is connected with investigation of arithmetical properties of rings, monoids and po-groups and it has its motivation in the theory of algebraic numbers. Various embedding theorems not only for po-groups, but also for integral domains (e.g., embedding into the GCD domain) might serve as a basis for various concepts in arithmetical investigation of rings, po-groups and monoids.

The axiomatic concept of a domain with this embedding property was formulated firstly in the book of Borewicz and Šafarevič [3]. After then this axiomatic concept was firstly transformed onto semigroups with cancellation by Skula $[16,17]$ and then transformed and generalized onto po-groups and monoids by several authors (see $[2,6,7,8,13,14])$. Nevertheless in all these transformations and generalizations the principal structure of this axiomatic concept remains the same and for po-groups it could be expressed as follows.

A po-group $G$ is said to admits some "divisor theory" if there exists an l-group $\Gamma$ and a homomorphism $h: G \longrightarrow \Gamma$ such that

(1) $h$ is an ordered embedding (i.e., $o$-monomorphism),

(2) $h(G)$ is dense in $\Gamma$ (in some specific sense).

For example, during the history of development of this problem the following principal formulations of the density condition (2) appeared, most of them being due to Skula, Arnold and Borewicz and Šafarevič.

$$
\begin{aligned}
& (\forall \alpha \in \Gamma) \quad \exists g_{1}, \ldots, g_{n} \in G, \quad \alpha=h\left(g_{1}\right) \wedge \cdots \wedge h\left(g_{n}\right), \\
& (\forall \alpha \in \Gamma) \quad \alpha=\bigwedge\{h(g): h(g) \geq \alpha\},
\end{aligned}
$$




$$
\begin{array}{ll}
(\forall \alpha, \beta \in \Gamma) \quad\{g \in G: h(g) \geq \alpha\}=\{g \in G: h(g) \geq \beta\} \Longrightarrow \alpha=\beta, \\
(\forall \alpha, \beta \in \Gamma) \quad(\exists \gamma \in \Gamma) \text { such that } \alpha \wedge \gamma=1, \beta \cdot \gamma \in h(G) .
\end{array}
$$

In po-groups and integral domains theory these conditions (1.1), (1.2), (1.3), and (1.4) characterize po-groups with various types of divisor theory or integral domains which are generalizations of Krull domains (see [2, 7, 13, 16]). For example, if $\Gamma$ is required to be a free abelian group $\mathbb{Z}^{(P)}$ for some set $P$ then the conditions (1.1), (1.2), and (1.3) are equivalent and $h$ is then called the theory of divisors of $G$. For general l-group $\Gamma$ the condition (1.1) characterizes po-groups with the so-called theory of quasi divisors (this notion was introduced by Aubert [2], although Jaffard investigated properties of such groups more early [9]), and the condition (1.4) leads to po-groups with the strong theory of quasi divisors (see [14]). (This last axiom was originaly formulated by Arnold [1] and later used by Skula [16].)

Although the conditions (1.2) and (1.3) were not investigated for general l-group separately, Aubert [2] mistakes (1.1) and (1.3) (and, hence (1.4)) for equivalent. This mistake was pointed out by Lucius [10] who proved (in language of integral domains) that (1.3) does not imply (1.1), in general, and he introduced (again in the language of integral domains) a new notion of a po-group with the GCD theory as a po-group $G$ with the embedding $h: G \longrightarrow \Gamma$ which satisfies the condition (1.3). He proved also that the group of divisibility of an integral domain $R$ satisfies the condition (1.3) if and only if $R$ is $v$-domain on the contrary to the result of Geroldinger and Močkoř [7] stating that the group of divisibility of $R$ satisfies the condition (1.1) if and only if $R$ is a Prüfer $v$-multiplication domain. Hence, po-groups which satisfy the condition (1.3) really represent a generalization of po-groups with quasi divisor theory.

In this paper, we want to describe some properties of po-groups with GCD theory. The principal problem we want to solve concerns the uniqueness of the GCD theory. It is well known (and for the first time it was proved by Jaffard [9]) that up to $o$-isomorphism for any po-group there exist as most one quasi divisor theory. This uniqueness property is closely connected with the Lorenzen $t$-group $\Lambda_{t}(G)$ of $G$, since for any quasi divisor theory $h: G \longrightarrow \Gamma$ the natural embedding $G \rightarrow \Lambda_{t}(G)$ is the theory of quasi divisors, as well, and $\Lambda_{t}(G)$ is $o$-isomorphic to $\Gamma$. For GCD theory $h: G \longrightarrow \Gamma$ the situation is a little more complicated. Although the embedding $G \longrightarrow \Lambda_{t}(G)$ is a GCD theory (in case that $G$ admits the GCD theory), as well, it is the open problem stated by Lucius [10] whether $\Lambda_{t}(G)$ is again (up to $o$-isomorphism) unique within l-groups $\Gamma$ with the GCD theory $h: G \longrightarrow \Gamma$. In this paper, we show that, in general, the GCD theory is not unique which answers the Lucius question in negative. We further introduce the notion of a complete GCD theory and we prove that $G$ admits the complete GCD theory if and only if it admits a GCD theory $h: G \longrightarrow \Gamma$ such that $\Gamma$ is Archimedean. Moreover, we show that a po-group admits the complete GCD theory if and only if any $v$-ideal is $v$-invertible and we prove that a complete GCD theory is at most unique.

2. Basic properties of groups with GCD theory. The notion of GCD theory was introduced by Lucius for integral domains only. We present firstly the translation of his definition for po-groups. 
DEFINITION 2.1. Let $G$ be a po-group. By a GCD theory of $G$ we mean a homomorphism $h$ of $G$ into an abelian l-group $\Gamma$ such that

(1) $h$ is an $o$-monomorphism,

(2) $(\forall \alpha, \beta \in \Gamma) \alpha=\beta \Leftrightarrow\{g \in G: h(g) \geq \alpha\}=\{g \in G: h(g) \geq \beta\}$.

It is clear that if $G$ admits a quasi divisor theory it admits the GCD theory, as well. In fact, let $h: G \longrightarrow \Gamma$ be the quasi divisor theory of $G$ and let $A=\{g \in G: h(g) \geq$ $\alpha\}=\{g \in G: h(g) \geq \beta\}=B$ for $\alpha, \beta \in \Gamma$. Since $\alpha=h\left(a_{1}\right) \wedge \cdots \wedge h\left(a_{n}\right), \beta=h\left(b_{1}\right) \wedge$ $\cdots \wedge h\left(b_{m}\right)$ for some $a_{i}, b_{j} \in G$, we have $a_{i} \in B$ and $b_{j} \in A$ for all $i, j$ and it follows that $\alpha=\beta$.

The principal tool for investigation of properties of po-groups with GCD theory seems to be the notion of an $r$-ideal. Recall that by an $r$-system of ideals in a po-group $G$ we mean a map $X \mapsto X_{r}\left(X_{r}\right.$ is called an $r$-ideal) from the set of all lower bounded subsets $X$ of $G$ into the power set of $G$ which satisfies the following conditions:

(1) $X \subseteq X_{r}$,

(2) $X \subseteq Y_{r} \Longrightarrow X_{r} \subseteq Y_{r}$,

(3) $\{a\}_{r}=a \cdot G^{+}=(a)_{r} \forall a \in G$,

(4) $a \cdot X_{r}=(a \cdot X)_{r} \forall a \in G$.

Recall that an $r$-system is called a $v$-system, if

$$
X_{v}=\bigcap_{X \subseteq(y)_{r}, y \in G}(y)_{r},
$$

and it is called a $t$-system, if

$$
X_{t}=\bigcup_{Y \subseteq X, Y \text { finite }} Y_{v}
$$

An $r$-system $r$ is said to be of a finite character, if $X_{r}=\bigcup_{Y \subseteq X, Y \text { finite }} Y_{r}$. An $r$-ideal $X_{r}$ is finitely generated if $X_{r}=Y_{r}$ for some finite subset $Y$. Clearly, any $t$-system is of finite character. On the set $\mathscr{I}_{r}(G)$ of $r$-ideals we may define an ordering by $X_{r} \leq Y_{r}$ if and only if $Y_{r} \subseteq X_{r}$ and a multiplication $X_{r} \times Y_{r}=\left(X_{r} Y_{r}\right)_{r}=(X \cdot Y)_{r}$. An $r$-ideal $A_{r}$ is called $r$-invertible if it has the inverse in the semigroup $I_{r}(G)$. A po-group $G$ with an $r$-system $r$ is $r$-closed if $\left(X_{r}: X_{r}\right) \subseteq G^{+}$for any $X_{r}$. For any $r$-closed po-group $G$ we may construct another $r$-system in $G$, denoted by $r_{a}$, such that $r_{a}$ is regularly closed, i.e., in the semigroup $\left(\mathscr{I}_{r}^{f}(G), \times\right)$ of all finitely generated $r$-ideals the cancellation law holds. The quotient group $\Lambda_{r}(G)$ of $\oiint_{r}^{f}(G)$ is called the Lorenzen $r$-group of $G$ and a map $h: G \longrightarrow \Lambda_{r}(G)$ defined by $h(g)=(g)_{r}$ is an $o$-isomorphism into. For $t$ and $v$ systems we have $t_{a}=t, v_{a}=v$ and the Lorenzen $r$-group for $r=t$ or $r=v$ then consists of all formal quotients $A_{r} / B_{r}$, where $A$ and $B$ are finite sets. A homomorphism $f:(G, r) \longrightarrow(H, s)$ of po-groups with ideal systems $r$ and $s$, respectively, is called $(r, s)$-morphism if for any lower bounded subset $X \subseteq G$ we have $f\left(X_{r}\right) \subseteq(f(X))_{s}$.

The theory of $r$-ideals of po-groups seems to be a tool which enables us to establish relationships between arithmetical properties of integral domains and the theory of po-groups. There exists a lot of works justifying these processes, one of the last result in this direction being a paper of Geroldinger and the author (see [7]) where it is proved that properties of being a Prüfer $v$-multiplication domain, a domain of Krull type, or an independent domain of Krull type, are purely multiplicative ones and can be expressed by using $r$-ideals in the corresponding groups of divisibility. 
We will now investigate some simple properties of po-groups with GCD theory. The density property from Definition 2.1 is equivalent to some other conditions as the following proposition states, where we used the following notation. Let $h: G \longrightarrow \Gamma$ be an $o$-monomorphism. For $\alpha \in \Gamma$ we put $\bar{\alpha}^{h}=\bar{\alpha}=\{g \in G: h(g) \geq \alpha\}=h^{-1}\left((\alpha)_{v} \cap\right.$ $h(G)$ ). For any subset $X$ in a po-group $G$ we write $g \leq X$ for $g \leq x$ for all $x \in X$ and by $\wedge X$ we understand $\wedge_{x \in X} x$.

Proposition 2.2. Let $G$ be a po-group and let $h: G \longrightarrow \Gamma$ be an o-monomorphism into an l-group $\Gamma$. Then the following statements are equivalent.

(1) $(\forall \alpha, \beta \in \Gamma) \bar{\alpha}=\bar{\beta} \Leftrightarrow \alpha=\beta$,

(2) $(\forall \alpha, \beta \in \Gamma) \bar{\alpha} \subseteq \bar{\beta} \Leftrightarrow \beta \leq \alpha$,

(3) $(\forall \alpha \in \Gamma) \alpha=\bigwedge h(\bar{\alpha})$,

(4) $(\forall \alpha \in \Gamma)(\alpha)_{v}=\left(h(G) \cap(\alpha)_{v}\right)_{v}$,

(5) $(\forall \alpha \in \Gamma)\left(\exists A_{v} \in \Phi_{v}(G)\right) \alpha=\bigwedge h\left(A_{v}\right)$,

(6) $(\forall \alpha \in \Gamma)(\exists A \subseteq G) A$ is lower bounded and $\alpha=\bigwedge h(A)$,

(7) $(\forall \alpha \in \Gamma)\left(\exists A_{t} \in \mathscr{I}_{t}(G)\right) \alpha=\bigwedge h\left(A_{t}\right)$.

For the proof of this proposition we need the following lemma.

LEMMA 2.3. Let $h: G \longrightarrow \Gamma$ be an o-monomorphism into an l-group $\Gamma$ such that it satisfies the condition (2) from Proposition 2.2. Then $h$ is a $(t, t)$-morphism.

Proof. Let $A \subseteq G$ be lower bounded and let $g \in A_{t}$. Then there exists a finite subset $K \subseteq A$ such that $g \in K_{t}=K_{v}$ and we have $h(g) \in(h(K))_{v}=(\bigwedge h(K))_{v}$. In fact, we show that $\overline{(\bigwedge h(K))^{-1}} \subseteq \overline{h(g)^{-1}}$. Let $y^{-1} \in \overline{(\bigwedge h(K))^{-1}}$. Then we have $h(y) \leq \wedge h(K) \leq$ $h(K)$ and it follows that $y \leq K$. Since $g \in K_{t}$, we have $g \geq y$ and it follows that $y^{-1} \in \overline{h(g)^{-1}}$. Then according to the condition (2), $h(g) \geq \bigwedge h(K)$ and $h(g) \in(h(K))_{v}$. Therefore, $h$ is a $(t, t)$-morphism.

Proof of Proposition 2.2. (1) $\Rightarrow(2)$. Let $\bar{\alpha} \subseteq \bar{\beta}$. We show that in this case $\overline{\alpha \vee \beta}=$ $\bar{\alpha}$. Clearly, $\overline{\alpha \vee \beta} \subseteq \bar{\alpha}$. For $g \in \bar{\alpha} \subseteq \bar{\beta}$ it follows that $h(g) \geq \alpha \vee \beta$ and $\bar{\alpha} \subseteq \overline{\alpha \vee \beta}$. From (1) then follows $\alpha \geq \beta$.

(2) $\Rightarrow(3)$. Let $\alpha \in \Gamma$. We show firstly that $(\alpha)_{v} \cap h(G) \neq \varnothing$. In fact, let us consider the following only possible cases.

(a) $\alpha \geq 1_{\Gamma}$. Assume that the statement is not true. Then $\left(\alpha^{2}\right)_{v} \cap h(G)=\varnothing$ and it follows that $\bar{\alpha}=\overline{\alpha^{2}}$. Therefore, we have $\alpha=\alpha^{2}$ according to (2) and it follows that $\alpha=1_{\Gamma}$. But in this case we have $h\left(1_{G}\right) \in\left(1_{\Gamma}\right)_{v} \cap h(G)$, a contradiction.

(b) $\alpha<1_{\Gamma}$. Then we have $h\left(1_{G}\right) \in\left(1_{\Gamma}\right)_{v} \cap h(G)$.

(c) $\alpha \| 1_{\Gamma}$. Since $\Gamma$ is directed, there exists $\beta \in \Gamma_{+}$such that $\alpha \cdot \beta \geq 1_{\Gamma}$ and it follows that $h(g) \in(\alpha \cdot \beta)_{v} \cap h(G)$ for some $g \in G$. Hence $h(g) \geq \alpha \cdot \beta \geq \alpha$.

Now let $\beta \in \Gamma$ be such that $\beta \leq h(\bar{\alpha})$. It follows that $\bar{\alpha} \subseteq \bar{\beta}$ and according to (2) we have $\alpha \geq \beta$. Therefore, $\alpha=\bigwedge(h(\bar{\alpha}))$.

$(3) \Rightarrow(4)$. Let $\alpha \in \Gamma$. Since $h(\bar{\alpha})$ is lower bounded by $\alpha$, there exists the $v$-ideal $(h(\bar{\alpha}))_{v}$. We have to prove only that $\alpha \in(h(\bar{\alpha}))_{v}$. Let $\beta \leq h(\bar{\alpha})$. From (3) it follows that $\beta \leq \alpha$ and we have $\alpha \in(h(\bar{\alpha}))_{v}$.

$(4) \Rightarrow(1)$. Let $\bar{\alpha}=\bar{\beta}$. Then $h(\bar{\alpha})=h(\bar{\beta})$ and according to $(4)$ it follows that $(\alpha)_{v}=(\beta)_{v}$. Hence, $\alpha=\beta$. 
(4) $\Rightarrow(5)$. Let $\alpha \in \Gamma$. Then $\bar{\alpha}$ is lower bounded in $G$. In fact, since the statement (2) holds, analogously as we did in the proof of implication $(2) \Rightarrow(3)$ we can prove that $h\left(g^{-1}\right) \in\left(\alpha^{-1}\right)_{v} \cap h(G)$ for some $g \in G$. Hence, $h(g) \leq \alpha$ and it follows that $g \leq \bar{\alpha}$ in $G$. Since $(4) \Leftrightarrow(3)$, we have $\alpha=\bigwedge h(\bar{\alpha})$. We prove that $\alpha=\bigwedge h\left(\bar{\alpha}_{v}\right)$. In fact, since $\bar{\alpha} \subseteq \bar{\alpha}_{v}$, then any lower bound of $\bar{\alpha}_{v}$ is less than or equal to $\alpha$. It remains to show that $\alpha \leq h\left(\bar{\alpha}_{v}\right)$. Let $g \in \bar{\alpha}_{v}$ and let $h(y) \leq \alpha$, hence, $\bar{\alpha} \subseteq(y)_{v}$. Since $g \in \bar{\alpha}_{v}$, then $g$ is contained in any principal $v$-ideal containing $\bar{\alpha}$, hence, we have $g \geq y$. Therefore, $h(y) \leq \alpha \wedge h(g)$ and it follows that $\overline{\alpha^{-1}} \subseteq \overline{(\alpha \wedge h(g))^{-1}}$. Since the opposite inclusion holds and (4) $\Leftrightarrow(1)$, we have $\alpha \leq h(g)$ and $\alpha \leq h\left(\bar{\alpha}_{v}\right)$.

$(5) \Rightarrow(6)$. Trivial.

(6) $\Rightarrow(3)$. Let $A$ be a lower bounded subset in $G$ and let $\alpha=\bigwedge h(A)$. Then $A \subseteq \bar{\alpha}$. We show that $\alpha=\wedge h(\bar{\alpha})$. Let $\beta \leq h(\bar{\alpha})$, then $\beta \leq h(A)$ and we have $\beta \leq \alpha$. Hence, $\alpha=\bigwedge h(\bar{\alpha})$.

(6) $\Rightarrow(7)$. Let $\alpha \in \Gamma$ and let $A \subseteq G$ be a lower bounded subset such that $\alpha=\bigwedge h(A)$. We prove that $\alpha=\bigwedge h\left(A_{t}\right)$. It is clear that for any lower bound $\beta$ of $h\left(A_{t}\right), \beta$ is a lower bound of $h(A)$ and it follows that $\beta \leq \alpha$. Hence, we have to prove only that $\alpha \leq h\left(A_{t}\right)$. Let $g \in A_{t}$. Then there exists a finite subset $K \subseteq A$ such that $g \in K_{t}$ and for $\beta=\bigwedge h(K) \in \Gamma$ we have $h(g) \in h\left(K_{t}\right) \subseteq(h(K))_{t}=(\beta)_{t}$, according to the lemma. Hence, $h(g) \geq \beta \geq \alpha=\bigwedge h(A)$.

(7) $\Rightarrow$ (3). Let $\alpha=\bigwedge h\left(A_{t}\right)$ for some lower bounded subset $A \subseteq G$. Then $A_{t} \subseteq \bar{\alpha}$ and it follows that $\alpha=\bigwedge h\left(A_{t}\right) \geq \bigwedge h(\bar{\alpha}) \geq \alpha$. Therefore, $\alpha=\bigwedge h(\bar{\alpha})$.

The following proposition has its origin in the paper of Clifford [4] who originaly proved that for a theory of divisors $h: G \longrightarrow \mathbb{Z}^{(P)}$ the set $\bar{\alpha}$ is $v$-ideal for any $\alpha \in$ $\mathbb{Z}^{(P)}$. In language of integral domains this proposition was proved for GCD theory by Lucius [10]. Due to the importance of this result for our next results we translate this statement into the language of po-groups and present a little different proof, although the proof is, in fact, contained in the proof of Proposition 2.2.

Proposition 2.4. Let $h: G \longrightarrow \Gamma$ be a GCD theory. Then for any $\alpha \in \Gamma$ and any lower bounded subset $A \subseteq G$ such that $\alpha=\bigwedge h(A)$ we have $\bar{\alpha}=A_{v}$.

Proof. We show firstly that $\bar{\alpha}$ is an $v$-ideal. From the proof of Proposition 2.2 it follows that $\bar{\alpha}$ is lower bounded in $G$. Let $g \in \bar{\alpha}_{v}$. Then according to Proposition 2.2,

$$
g \in \bar{\alpha}_{v}=\bigcap_{\substack{y \in G \\ \bar{\alpha} \subseteq(y)_{v}=\overline{h(y)}}}(y)_{v}=\bigcap_{\substack{y \in G \\ \alpha \geq h(y)}}(y)_{v} .
$$

Then $\overline{\alpha^{-1}}=\overline{(h(g) \wedge \alpha)^{-1}}$. In fact, let $y \in \overline{\alpha^{-1}}$. Then $\alpha \geq h\left(y^{-1}\right)$ and we have $g \in$ $\left(y^{-1}\right)_{v}$. Thus, $h\left(y^{-1}\right) \leq \alpha \wedge h(g)$ and it follows that $y \in \overline{(\alpha \wedge h(g))^{-1}}$. The other inclusion is trivial. Then from Proposition 2.2 then it follows that $\alpha \leq h(g)$ and $g \in \bar{\alpha}$. Therefore, $\bar{\alpha}=\bar{\alpha}_{v}$. Now let $\alpha=\bigwedge h(A)$. Then $A \subseteq \bar{\alpha}$ and $A_{v} \subseteq \bar{\alpha}_{v}=\bar{\alpha}$. Conversely, for $g \in \bar{\alpha}$ and for any $y \leq A$ we have $h(y) \leq \alpha \leq h(g)$ and it follows that $y \leq g$. Hence, $g \in A_{v}$ and $A_{v}=\bar{\alpha}$.

The following proposition is a generalization of the above-mentioned result of Clifford. 
Proposition 2.5. Let $h: G \longrightarrow \Gamma$ be a GCD theory. Then the map ${ }^{-}:(\Gamma, \cdot) \longrightarrow$ $\left(\Phi_{v}(G), \times\right)$ such that $\alpha \mapsto \bar{\alpha}$ is an o-isomorphism into and the following diagram commutes:

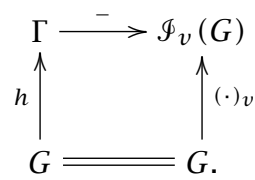

Proof. Let $\alpha, \beta \in \Gamma$. Then according to Proposition 2.2, we have $\alpha \cdot \beta=\wedge h(\overline{\alpha \cdot \beta})$. On the other hand, we obtain

$$
\alpha \cdot \beta=(\bigwedge h(\bar{\alpha})) \cdot(\bigwedge h(\bar{\beta}))=\bigwedge h(\bar{\alpha} \cdot \bar{\beta}) .
$$

Then according to Proposition 2.4, we have

$$
\begin{aligned}
& \bar{\alpha} \times \bar{\beta}=\bar{\alpha}_{v} \times \bar{\beta}_{v}=\bigwedge h(\bar{\alpha} \cdot \bar{\beta}), \\
& \overline{\alpha \cdot \beta}=(\overline{\alpha \cdot \beta})_{v}=\bigwedge h(\overline{\alpha \cdot \beta}) .
\end{aligned}
$$

Therefore, $\overline{\alpha \cdot \beta}=\bar{\alpha} \times \bar{\beta}$ and ${ }^{-}$is a group homomorphism. Moreover, from Proposition 2.2 it follows that it is injective and $o$-isomorphism into. The commutativity of the diagram follows from $(g)_{v}=\overline{h(g)}$.

The following corollary (in integral domains language) is due to Lucius [10].

COROLLARY 2.6. Let $h: G \longrightarrow \Gamma$ be a GCD theory. Then any finitely generated $v$-ideal of $G$ is $v$-invertible.

Proof. Let $g_{1}, \ldots, g_{n} \in G$ and let $\alpha=h\left(g_{1}\right) \wedge \cdots \wedge h\left(g_{n}\right)$. Then according to Proposition 2.4, we have $\bar{\alpha}=\left(g_{1}, \ldots, g_{n}\right)_{v}$ and it follows that

$$
1_{g_{v}(G)}=\left(1_{G}\right)_{v}=\overline{\alpha \cdot \alpha^{-1}}=\bar{\alpha} \times \overline{\alpha^{-1}} .
$$

Hence, $\bar{\alpha}$ is $v$-invertible.

Lucius pointed out that integral domain with GCD theory need not be Prüfer $v$ multiplication domain. For this he used the example of $v$-domain which is not Prüfer $v$-multiplication domain constructed in [11]. The following lemma enables us to use this example to show that po-groups with GCD theory need not admit quasi divisor theory.

LEMMA 2.7. Let $R$ be an integral domain and let $G(R)$ be its group of divisibility. Then there exists an isomorphism $\sigma:\left(\mathscr{I}_{v}(G(R)), \times\right) \longrightarrow\left(\mathscr{I}_{v}(R), \times\right)$ such that $\sigma\left(g_{v}^{f}(G(R))\right)=و_{v}^{f}(R)$.

Proof. Let $K$ be the quotient field of $R$ and let $w: K^{\times} \longrightarrow G(R)$ be the corresponding semi-valuation of $R$. For $A \in \Phi_{v}(G(R))$ we set $\sigma(A)=w^{-1}(A) \cup\left\{0_{K}\right\}$. Let $a, b \in \sigma(A)$. Since $w(a+b) \geq \alpha$ for all $\alpha \in G(R)$ such that $\alpha \leq w(a), w(b)$ (see [15]), we have $w(a+b) \in(w(a), w(b))_{v} \subseteq A_{v}$ and it follows that $\sigma(A)$ is an ideal. It is clear that $\sigma$ is a homomorphism. Conversely, for $J \in \mathscr{I}_{v}(R)$ we set $\psi(J)=w\left(J^{\times}\right)$. Then $\psi(J)$ is $v$-ideal and $\psi$ is a homomorphism which is inverse to $\sigma$. 
Let $R$ be a $v$-domain which is not Prüfer $v$-multiplication domain. Then according to previous lemma every finitely generated $v$-ideal of $G=G(R)$ is $v$-invertible, but finitely generated $v$-ideal of $G$ do not form a group. Hence, $G$ does not admit quasi divisor theory according to [2] or [7]. On the other hand, $G$ admits GCD theory as the following proposition shows (a version of this proposition for integral domains was proved by Lucius [10]).

Proposition 2.8. Let $G$ be a po-group and let any finitely generated $v$-ideal of $G$ be $v$-invertible. Then the embedding $h: G \longrightarrow \Lambda_{v}(G)$ is the GCD theory of $G$.

Proof. It should be observed that the Lorenzen group $\Lambda_{v}(G)$ exists, since $G$ is $v$ closed. Moreover, $\Lambda_{v}(G)=\Lambda_{t}(G)$ in this case and elements of $\Lambda_{v}(G)$ can be identified with some $v$-ideals of $G$. In fact, for any formal fraction $A_{v} / B_{v} \in \Lambda_{v}(G)$ (with $A, B$ finite) there exists $v$-ideal $C_{v}$ such that $A_{v}=B_{v} \times C_{v}$. In this case, $A_{v} / B_{v}$ can be identified with $C_{v}$. Hence, let $C_{v}, D_{v}$ be such that $\overline{C_{v}}=\overline{D_{v}}$, where $\overline{X_{v}}=\left\{g \in G:(g)_{v} \subseteq\right.$ $\left.X_{v}\right\}$. But in this case $C_{v}=D_{v}$ and $(\cdot)_{v}: G \longrightarrow \Lambda_{v}(G)$ is the GCD theory.

In quasi divisor theory it is well known that the embedding $G \rightarrow \Lambda_{t}(G)$ is (up to isomorphisms) the only possible quasi divisor theory (if it exists). In the next section, we show that for GCD theory it is not true. This answers in negative the question presented by Lucius [10].

Proposition 2.9. Let $\sigma: G \longrightarrow G^{\prime}$ be a surjective $(v, v)$-morphism of po-groups. Then if $G$ admits a GCD theory the same is true for $G^{\prime}$.

Proof. Let $X \subseteq G^{\prime}$ be a finite set and let $A \subseteq G$ be a finite set such that $\sigma(A)=X$. Let $C_{v}$ be the $v$-inverse of $A_{v}$ in $G,\left(1_{G}\right)_{v}=(A \cdot C)_{v}$. Then we have

$$
1_{G^{\prime}} \in \sigma\left(\left(1_{G}\right)_{v}\right)=\sigma\left((A \cdot C)_{v}\right) \subseteq(\sigma(A \cdot C))_{v}=X_{v} \times(\sigma(C))_{v}
$$

Thus, $\left(1_{G^{\prime}}\right)_{v} \subseteq X_{v} \times(\sigma(C))_{v}$. Since $A \cdot C \subseteq\left(1_{G}\right)_{v}$, we have $X \cdot \sigma(C) \subseteq \sigma\left(\left(1_{G}\right)_{v}\right) \subseteq$ $\left(1_{G^{\prime}}\right)_{v}$ and it follows that $X_{v} \times(\sigma(C))_{v} \subseteq\left(1_{G^{\prime}}\right)_{v}$. Therefore, $X_{v}$ is $v$-invertible in $G^{\prime}$ and $G^{\prime}$ admits a GCD according to Proposition 2.8.

For any embedding $h: G \longrightarrow \Gamma$ of a po-group $G$ into an l-group we can construct the divisor class group $\mathscr{C}_{h}=\Gamma / h(G)$ of this embedding. Hence, we have the following short exact sequence

$$
1-\longrightarrow G \stackrel{h}{\longrightarrow} \Gamma \stackrel{\varphi_{h}}{\rightarrow} \mathscr{C}_{h}-\longrightarrow 0 .
$$

For po-groups with quasi divisor theory this divisor class group has special importance, since the density condition of $h$ can be substitute by some density condition of $\varphi_{h}$ (see $[12,16]$ ). In the following proposition, we show that even for po-groups with GCD this short exact sequence has some density property.

Proposition 2.10. Let $h: G \longrightarrow \Gamma$ be a $G C D$ theory. Then for any $\alpha \in \Gamma, \alpha>1$, we have

$$
\varphi_{h}\left(\Gamma \backslash(\alpha)_{v}\right)=\mathscr{C}_{h}
$$


Proof. We show firstly that for any $\beta \in \Gamma$ there exists $\gamma \in \Gamma_{+}$such that $\varphi_{h}(\beta)=$ $\varphi_{h}(\gamma)$. In fact, according to the proof of Proposition 2.2, $\left(\beta^{-1}\right)_{v} \cap h(G) \neq \varnothing$ and $\gamma=$ $\beta \cdot h(g) \geq 1$ for some $g \in G$. Hence $\varphi_{h}(\beta)=\varphi_{h}(\gamma)$.

Let $\varphi_{h}(\beta) \in \mathscr{C}_{h}$. Then from the above statement if follows that $-\varphi_{h}(\beta) \in \varphi_{h}\left(\Gamma_{+}\right)$ and there exists $\delta \in \Gamma_{+}$such that $-\varphi_{h}(\beta)=\varphi_{h}(\delta)$. Moreover, there exists $\gamma \in \Gamma \backslash(\alpha)_{v}$ such that $\delta \cdot \gamma \in h(G)$. In fact, let us consider the following only possible cases.

(a) $\alpha \| \delta$ or $\alpha>\delta$. Then from Proposition 2.2 it follows that $h(\bar{\delta}) \backslash h(\bar{\alpha}) \neq \varnothing$. Let $h(g) \in h(\bar{\delta}) \backslash h(\bar{\alpha})$. Then $h(g) \notin(\alpha)_{v}$ and there exists $\gamma \in \Gamma_{+}$such that $h(g)=\delta \cdot \gamma$ and $\gamma \in \Gamma_{+} \backslash(\alpha)_{v}$.

(b) $\alpha \leq \delta$. Then $\alpha \cdot \delta>\delta \geq \alpha$ and $h(\overline{\alpha \cdot \delta}) \subset h(\bar{\delta})$. In fact, if the inclusion is not proper, we have $\alpha \cdot \delta=\bigwedge h(\overline{\alpha \cdot \delta})=\bigwedge h(\bar{\delta})=\delta$ according to Proposition 2.2, a contradiction. Let $h(g) \in h(\bar{\delta}) \backslash h(\overline{\alpha \cdot \delta})$. Then $h(g)=\gamma \cdot \delta$ for some $\gamma \geq 1$. If $\gamma \geq \alpha$, then we have $h(g) \geq \alpha \cdot \delta$, a contradiction. Hence, $\gamma \in \Gamma_{+} \backslash(\alpha)_{v}$.

From this property we obtain $\varphi_{h}(\delta)+\varphi_{h}(\gamma)=0$ and $\gamma \in \Gamma_{+} \backslash(\alpha)_{v}$. Hence, $\varphi_{h}\left(\Gamma_{+}\right) \subseteq$ $\varphi_{h}\left(\Gamma_{+} \backslash(\alpha)_{v}\right)$.

3. Groups with complete GCD theories. For a GCD theory $h: G \longrightarrow \Gamma$ the set $\Phi_{v}(G)$ of all $v$-ideals of $G$ has a special subset, namely $\{\bar{\alpha}: \alpha \in \Gamma\} \subseteq I_{v}(G)$, as follows from Proposition 2.4. A natural question is when this subset equals to $\Phi_{v}(G)$. This problem is solved by the following proposition.

Proposition 3.1. Let $h: G \longrightarrow \Gamma$ be a GCD theory. Then the following statements are equivalent.

(1) For any $v$-ideal $A_{v}$ in $G$ there exists $\alpha \in \Gamma$ such that $A_{v}=\bar{\alpha}$.

(2) For any lower bounded subset $A \subseteq G$ there exists $\bigwedge h(A)$ in $\Gamma$.

(3) $\Gamma$ is a complete l-group.

Proof. (2) $\Rightarrow(1)$. Let $A_{v} \in \Phi_{v}(G)$ and let $\alpha=\bigwedge h(A)$. Then $A \subseteq \bar{\alpha}$ and since $\bar{\alpha}$ is $v$-ideal in $G$, we have $A_{v} \subseteq \bar{\alpha}$. Let $g \in \bar{\alpha}$ and let $b$ be a lower bound of $A$. Then $h(g) \geq \alpha \geq h(b)$ and we have $g \geq b$. Therefore, $g \in A_{v}$ and $A_{v}=\bar{\alpha}$.

(1) $\Rightarrow(3)$. Let $\Phi \subseteq \Gamma$ be a lower bounded subset and let $\beta \leq \Phi$ for some $\beta \in \Gamma$. For any $\alpha \in \Phi$ we have $\alpha=\bigwedge h(\bar{\alpha})$ according to Proposition 2.2. Then $\bigcup_{\alpha \in \Phi} \bar{\alpha}$ is lower bounded in $G$. In fact, according to Proposition 2.2, $\beta^{-1}=\bigwedge h\left(\overline{\beta^{-1}}\right) \leq h\left(r^{-1}\right)$ for some $r^{-1} \in \overline{\beta^{-1}}$. Then for any $g \in \bigcup_{\alpha \in \Phi} \bar{\alpha}$ we have $h(g) \geq \alpha \geq \beta \geq h(r)$ and $r$ is a lower bound of $\bigcup_{\alpha \in \Phi} \bar{\alpha}$. Let $A_{v}=\left(\bigcup_{\alpha \in \Phi} \bar{\alpha}\right)_{v}$. Then according to (1), there exists $\gamma \in \Gamma$ such that $\bar{\gamma}=A_{v}$. We have $\gamma=\bigwedge \Phi$ in $\Gamma$. In fact, for any $\alpha \in \Phi$ we have $\bar{\alpha} \subseteq \bigcup_{\rho \in \Phi} \bar{\rho} \subseteq A_{v}=\bar{\gamma}$ and from Proposition 2.2 it follows that $\alpha \geq \gamma$. Further, let $\omega \leq \alpha$ for all $\alpha \in \Phi$. Then $\bar{\alpha} \subseteq \bar{\omega}$ and $\bigcup_{\alpha \in \Phi} \bar{\alpha} \subseteq \bar{\omega}$. Since $\omega$ is $v$-ideal, we have $\bar{\gamma}=A_{v} \subseteq \bar{\omega}$ and it follows that $\gamma \geq \omega$ by Proposition 2.2.

(3) $\Rightarrow(2)$. Trivial.

A GCD theory of a po-group $G$ that satisfies the equivalent conditions of Proposition 3.1 will be called a complete GCD theory. It should be observed that there are examples of GCD theory $h: G \longrightarrow \Gamma$ such that $\{\bar{\alpha}: \alpha \in \Gamma\} \subset \Phi_{v}(G)$. In fact, for any non complete l-group $\Gamma$ the identity map $\Gamma \longrightarrow \Gamma$ is such example, as follows from the previous proposition. 
COROLlary 3.2. Let $h: G \longrightarrow \Gamma$ be a complete GCD theory. Then $h$ is a $(v, v)$ morphism.

Proof. Let $A \subseteq G$ be a lower bounded set. Then for $\alpha=\bigwedge h(A)$ we have $h\left(A_{v}\right)=$ $h(\bar{\alpha}) \subseteq(\bigwedge h(A))_{v}=(\alpha)_{v}=(h(A))_{v}$ as follows from Proposition 3.1.

COROLlaRY 3.3. Let $h: G \longrightarrow \Gamma$ be a complete GCD theory. Then for any lower bounded subset $\Phi \subseteq \Gamma$ we have

$$
\overline{\bigwedge_{\alpha \in \Phi} \alpha}=\bigwedge_{\alpha \in \Phi} \bar{\alpha}
$$

Proof. For any $\beta \leq \Phi$ in $\Gamma$ there exists $b \in G$ such that $h(b) \leq \beta$. Then $b$ is a lower bound of $\bigcup_{\alpha \in \Phi} \bar{\alpha}$ and according to Proposition 3.1, there exists $\gamma \in \Gamma$ such that $\bar{\gamma}=\left(\bigcup_{\alpha \in \Phi} \bar{\alpha}\right)_{v}=\bigwedge_{\alpha \in \Phi} \bar{\alpha}$. We prove that $\gamma=\bigwedge_{\alpha \in \Phi} \alpha$. Since $\bar{\alpha} \subseteq \bar{\gamma}$, it follows that $\gamma \leq \alpha$ for any $\alpha \in \Phi$. For any $\omega \in \Gamma$ such that $\omega \leq \Phi$ we have $\bigcup_{\alpha \in \Phi} \bar{\alpha} \subseteq \bar{\omega}$ and since $\bar{\omega}$ is a $v$-ideal, we have $\bar{\gamma} \subseteq \bar{\omega}$. Hence, $\gamma \geq \omega$.

THEOREM 3.4. Let $G$ be a po-group. Then $G$ admits a complete GCD theory if and only if it admits a GCD theory $h: G \longrightarrow \Gamma$ such that $\Gamma$ is an Archimedean l-group.

Proof. Let $h$ be a GCD theory such that $\Gamma$ is Archimedean. It is well known (cf. [5]) that for any abelian Archimedean l-group $\Gamma$ its completion $\hat{\Gamma}$ can be constructed such that

(1) $\hat{\Gamma}$ is a complete l-group,

(2) $\Gamma$ is an l-subgroup of $\widehat{\Gamma}$,

(3) $\Gamma$ is dense in $\hat{\Gamma}$, i.e., for any $1<\mathbf{a} \in \hat{\Gamma}$ there exists $\alpha \in \Gamma$ such that $1<\alpha \leq \mathbf{a}$,

(4) For any $\mathbf{a} \in \hat{\Gamma}$ we have $\mathbf{a}=\bigwedge\{\beta \in \Gamma: \beta \geq \mathbf{a}\}$.

We observe further that if $\Delta \subseteq \Gamma$ is such that $\alpha=\wedge \Delta$ exists in $\Gamma$, then $\alpha$ is the infimum of $\Delta$ in $\hat{\Gamma}$, as well, i.e., we need not to distinguish infimum operations in $\Gamma$ and $\hat{\Gamma}$. In fact, let $\alpha=\wedge \Delta$ in $\Gamma$ and let $\mathbf{a} \in \hat{\Gamma}$ be such that $\mathbf{a} \leq \Delta$. Then we have $\alpha \vee \mathbf{a} \leq \Delta$ and we have to show that $\alpha \vee \mathbf{a}=\alpha$. Suppose, by the way of contradiction that $\alpha \vee \mathbf{a}>\alpha$. Then we have $1<(\alpha \vee \mathbf{a}) \cdot \alpha^{-1}$. Since $\Gamma$ is dense in $\hat{\Gamma}$, there exists $\beta \in \Gamma$ such that $1<\beta \leq(\alpha \vee \mathbf{a}) \cdot \alpha^{-1}$. Hence, for any $\omega \in \Delta$ we have $\omega \geq \alpha \vee \mathbf{a} \geq \beta \cdot \alpha>\alpha$, a contradiction.

Now, let $\mathbf{h}: G \stackrel{h}{\rightarrow} \Gamma \longrightarrow \widehat{\Gamma}$ be the composition of $h$ and the embedding of $\Gamma$ into $\widehat{\Gamma}$. We show that $\mathbf{h}$ is a complete GCD theory. Let $\mathbf{a} \in \hat{\Gamma}$. Then $\mathbf{a}=\bigwedge_{\beta \in \Phi} \beta$, where $\Phi=$ $\{\beta \in \Gamma: \beta \geq \mathbf{a}\}$. Since $h$ is a GCD theory, for any $\beta \in \Phi$ we have $\beta=\bigwedge h(\bar{\beta})=\bigwedge \mathbf{h}(\bar{\beta})$ and it follows that $\overline{\mathbf{a}}=\bigcup_{\beta \in \Phi} \bar{\beta}$. In fact, for $g \in \overline{\mathbf{a}}$ we have $\mathbf{h}(g) \geq \mathbf{a}$ and $\mathbf{h}(g) \in \Phi, g \in$ $\overline{\mathbf{h}(g)} \subseteq \bigcup_{\beta \in \Phi} \bar{\beta}$ (the other inclusion is trivial). Then we have $\mathbf{a}=\bigwedge \mathbf{h}(\overline{\mathbf{a}})=\bigwedge \mathbf{h}\left(\bigcup_{\beta \in \Phi} \bar{\beta}\right)$. In fact, let $\mathbf{b} \in \hat{\Gamma}$ be such that $\mathbf{b} \leq \mathbf{h}\left(\cup_{\beta \in \Phi} \bar{\beta}\right)$. Then $\mathbf{b} \leq \mathbf{h}(\bar{\beta})$ for any $\beta \in \Phi$ and we have $\mathbf{b} \leq \bigwedge \mathbf{h}(\bar{\beta})=\bigwedge h(\bar{\beta})=\beta$. Therefore, $\mathbf{b} \leq \bigwedge_{\beta \in \Phi} \beta=\mathbf{a}$ and $\mathbf{h}$ is a GCD theory according to Proposition 2.2.

Conversely, if $G$ admits a complete GCD theory $h: G \longrightarrow \Gamma$, then $\Gamma$ is a complete l-group and it follows that $\Gamma$ is Archimedean.

From this theorem it follows that, in general, GCD theory is not unique. In fact, let $h: G \longrightarrow \Gamma$ be a GCD theory that is not complete and let $\Gamma$ be an Archimedean lgroup. Then $h: G \longrightarrow \hat{\Gamma}$ is a (complete) GCD theory and $\Gamma$ is not isomorphic to $\hat{\Gamma}$. This answers in negative the question of Lucius [10]. 
Although for a GCD theory $h: G \longrightarrow \Gamma$ the inclusion relation $\{\bar{\alpha}: \alpha \in \Gamma\} \subseteq \mathscr{I}_{v}(G)$ can be a proper inclusion, the set of $v$-ideals of the form $\bar{\alpha}$ is even in this case dense in $\Phi_{v}(G)$. In the following proposition, we show that even for general GCD theory, $\Phi_{v}(G)$ represents a lattice completion of the set $\{\bar{\alpha}: \alpha \in \Gamma\}$. Moreover, from Proposition 2.5 it follows that $(\Gamma, \cdot, \leq)$ is isomorphic to $(\{\bar{\alpha}: \alpha \in \Gamma\}, \times, \leq)$ and from the next proposition it follows that $\mathscr{I}_{v}(G)$ represents the lattice completion of the lattice $(\Gamma, \wedge)$.

Proposition 3.5. Let $h: G \longrightarrow \Gamma$ be a GCD theory. Then the following statements hold.

(1) For any lower bounded subset $\phi \subseteq \Gamma$ there exists $\bigwedge_{\alpha \in \Phi} \bar{\alpha}$ in $\Phi_{v}(G)$.

(2) For any $v$-ideal $A_{v}$ in $G$ there exists a lower bounded subset $\Phi$ in $\Gamma$ such that $A_{v}=\bigwedge_{\alpha \in \Phi} \bar{\alpha}$.

Proof. (1) Let $\beta$ be a lower bound of $\Phi$. From Proposition 2.2 it follows that there exists $b \in G$ such that $h(b) \leq \beta$ and it follows that $b$ is a lower bound of $\bigcup_{\alpha \in \Phi} \bar{\alpha}$. Then it is clear that $\left(\bigcup_{\alpha \in \Phi} \bar{\alpha}\right)_{v}=\bigwedge_{\alpha \in \Phi} \bar{\alpha}$.

(2) Let $A_{v} \in I_{v}(G)$. We set $\Phi=\left\{\alpha \in \Gamma: A_{v} \subseteq \bar{\alpha}\right\}$. Then it is clear that $\Phi \neq \varnothing$ since, for example, for any finite $K \subseteq A$ we have $\bigwedge h(K) \in \Phi$. Then $\bigcup_{\alpha \in \Phi} \bar{\alpha} \subseteq A_{v}$ is lower bounded and we have $\bigwedge_{\alpha \in \Phi} \bar{\alpha}=\left(\bigcup_{\alpha \in \Phi} \bar{\alpha}\right)_{v}=A_{v}$. In fact, the inclusion $\subseteq$ is trivial. Let $a \in A_{v}$, then for any $g \in \overline{h(a)}$ we have $g \in(a)_{v} \subseteq A_{v}$ and it follows that $h(a) \in \Phi$. Therefore, $a \in \overline{h(a)} \subseteq\left(\bigcup_{\alpha \in \Phi} \bar{\alpha}\right)_{v}$.

Proposition 3.6. Let $h: G \longrightarrow \Gamma$ be a complete GCD theory. Then ${ }^{-}:(\Gamma, \cdot, \leq) \longrightarrow$ $\left(I_{v}(G), \times, \leq\right)$ is a complete l-isomorphism with the inverse l-isomorphism $X$ such that $\chi\left(A_{v}\right)=\bigwedge h\left(A_{v}\right)=\bigwedge h(A)$ for any $A_{v} \in \mathscr{I}_{v}(G)$.

Proof. From Proposition 2.5 it follows that ${ }^{-}$is an $o$-isomorphism into. Let $A_{v} \in$ $\Phi_{v}(G)$. Then from Proposition 3.5 and corollary of Proposition 3.1 it follows that $A_{v}=\bigwedge_{\alpha \in \Phi} \bar{\alpha}=\bigwedge_{\alpha \in \Phi} \alpha$. Hence, ${ }^{-}$is surjective and complete l-homomorphism. Further, we show that for any $A_{v} \in I_{v}(G), \wedge h\left(A_{v}\right)=\bigwedge h(A)$. In fact, let $\alpha=\bigwedge h(A)$, then according to Proposition 2.4, we have $\bar{\alpha}=A_{v}$. Hence, for any $a \in A_{v}$ we have $h(a) \geq \alpha$ and it follows that $\alpha=\bigwedge h(A) \geq \bigwedge h\left(A_{v}\right) \geq \alpha$. Hence, $x\left(A_{v}\right)=\bigwedge h\left(A_{v}\right)=\bigwedge h(A)$. Moreover, $x$ is a group homomorphism. In fact, we have

$$
\chi\left(A_{v} \times B_{v}\right)=\chi\left((A \cdot B)_{v}\right)=\bigwedge h(A \cdot B)=\bigwedge h(A) \cdot \bigwedge h(B)=\chi\left(A_{v}\right) \cdot \chi\left(B_{v}\right) .
$$

Further, $x$ and ${ }^{-}$are mutually inverse maps. In fact, for $\alpha \in \Gamma$ we have $\chi(\bar{\alpha})=\bigwedge h(\bar{\alpha})=$ $\alpha$ as follows from Proposition 2.2, and for $A_{v} \in \Phi_{v}(G)$ we have $\overline{\chi\left(A_{v}\right)}=\overline{\bigwedge h\left(A_{v}\right)}=$ $\overline{\bigwedge h(A)}=A_{v}$, according to Proposition 2.4. Finally, $\chi$ is a complete l-homomorphism. In fact, let $\mathscr{I} \subseteq \mathscr{I}_{v}(G)$ be a lower bounded subset and let $\Phi=\chi(\mathscr{I}) \subseteq \Gamma$. Then $\Phi$ is lower bounded and for $\alpha=\chi\left(A_{v}\right)$ we have $\bar{\alpha}=A_{v}$. Then we obtain

$$
\chi\left(\bigwedge_{A_{v} \in \mathscr{I}} A_{v}\right)=\chi\left(\bigwedge_{\alpha \in \Phi} \bar{\alpha}\right)=\chi\left(\overline{\bigwedge_{\alpha \in \Phi} \alpha}\right)=\bigwedge_{\alpha \in \Phi} \alpha=\bigwedge_{A_{v} \in \Phi} \chi\left(A_{v}\right)
$$

according to the corollary of Proposition 3.1. 
COROLLARY 3.7. Let $G$ be a po-group. Then $G$ admits a complete $G C D$ theory if and only if any $v$-ideal of $G$ is $v$-invertible.

Proof. Let $G$ admits a complete GCD theory. Then according to the previous Proposition 3.6, $\left(\Phi_{v}(G), \times\right)$ is a group. Conversely, let $\Phi_{v}(G)$ be a group. Then it is clear that it is a complete l-group, where for any lower bounded subset $\Phi \subseteq \Phi_{v}(G)$, the set $B=\bigcup_{A_{v} \in \Phi} A$ is lower bounded in $G$ and $B_{v}=\bigwedge_{A_{v} \in \Phi} A_{v}$. Moreover, for any $v$-ideal $A_{v}$ we have $A_{v}=\left(\bigcup_{a \in A}(a)_{v}\right)_{v}=\bigwedge_{a \in A}(a)_{v}$. Therefore, the embedding $(\cdot)_{v}$ : $G \longrightarrow \Phi_{v}(G)$ is a complete GCD theory according to Propositions 2.2 and 3.1.

COROLLARY 3.8. Any po-group $G$ admits at most (up to o-isomorphisms) one complete GCD theory.

Proof. Let $h: G \longrightarrow \Gamma$ be a complete GCD theory. Since $\Gamma$ is a complete l-group, it is Archimedean and according to Proposition 3.6, $\Gamma$ is $o$-isomorphic to $\Phi_{v}(G)$.

ACKNOWLEDGMENT. The research was partly supported by the GA ČR (201/97/ 0433).

\section{REFERENCES}

[1] I. Arnold, Ideale in kommutativen halbgruppen, Rec. Math. Soc. Math. Moscow 36 (1929), 401-407.

[2] K. E. Aubert, Divisors of finite character, Ann. Mat. Pura Appl. (4) 133 (1983), 327-361. MR 85f:06026. Zbl 533.20034.

[3] A. I. Borevich and I. R. Shafarevich, Number Theory, Academic Press, New York, 1966. MR 33\#4001. Zbl 145.04902.

[4] A. H. Clifford, Arithmetic and ideal theory of abstract multiplication, Ann. of Math. 39 (1938), 594-610.

[5] P. Conrad and D. McAlister, The completion of a lattice ordered group, J. Austral. Math. Soc. 9 (1969), 182-208. MR 40\#2585. Zbl 172.31601.

[6] A. Geroldinger and F. Halter-Koch, Realization theorems for semigroups with divisor theory, Semigroup Forum 44 (1992), no. 2, 229-237. MR 93c:20096. Zbl 751.20045.

[7] A. Geroldinger and J. Močkoř, Quasi-divisor theories and generalizations of Krull domains, J. Pure Appl. Algebra 102 (1995), no. 3, 289-311. MR 96k:13029. Zbl 853.13012.

[8] F. Halter-Koch, Ein Approximationssatz für Halbgruppen mit Divisorentheorie, Resultate Math. 19 (1991), no. 1-2, 74-82. MR 91m:13001. Zbl 742.20060.

[9] P. Jaffard, Les Systèmes D'idéaux, Dunod, Paris, 1960. MR 22\#5628. Zbl 101.27502.

[10] F. Lucius, Rings with a theory of greatest common divisors, Manuscripta Math. 95 (1998), no. 1, 117-136. MR 99b:13021. Zbl 901.13001.

[11] J. L. Mott and M. Zafrullah, On Prüfer v-multiplication domains, Manuscripta Math. 35 (1981), no. 1-2, 1-26. MR 83d:13026. Zbl 477.13007.

[12] J. Močkoř, Divisor class group and theory of quasi-divisors, to appear in Publ. Math. Debrecen.

[13] _ $t$-valuations and the theory of quasi-divisors, J. Pure Appl. Algebra 120 (1997), no. 1, 51-65. MR 98g:13021. Zbl 885.06008.

[14] J. Močkoř and A. Kontolatou, Groups with quasi-divisors theory, Comment. Math. Univ. St. Paul. 42 (1993), no. 1, 23-36. MR 94m:06018. Zbl 794.06015.

[15] J. Ohm, Semi-valuations and groups of divisibility, Canad. J. Math. 21 (1969), 576-591. MR 39\#4146. Zbl 177.06501.

[16] L. Skula, Divisorentheorie einer Halbgruppe, Math. Z. 114 (1970), 113-120. MR 41 \#7009. Zbl 185.04804. 
[17] On c-semigroups, Acta Arith. 31 (1976), no. 3, 247-257. MR 56\#3164. Zbl 338.13019 .

JiŘí MOČKOř: DEPARTMENT OF MATHEMATICS, UNIVERSITY OF OSTRAVA, CZ-702 00, OSTRAVA, CZECH REPUBLIC

E-mail address: Jiri.Mockor@osu.cz 


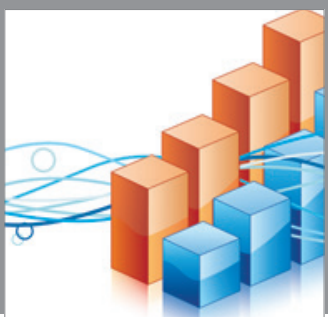

Advances in

Operations Research

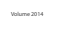

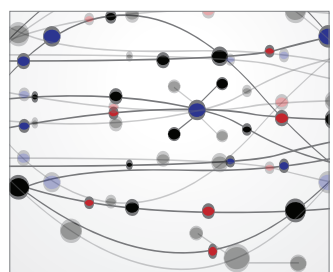

\section{The Scientific} World Journal
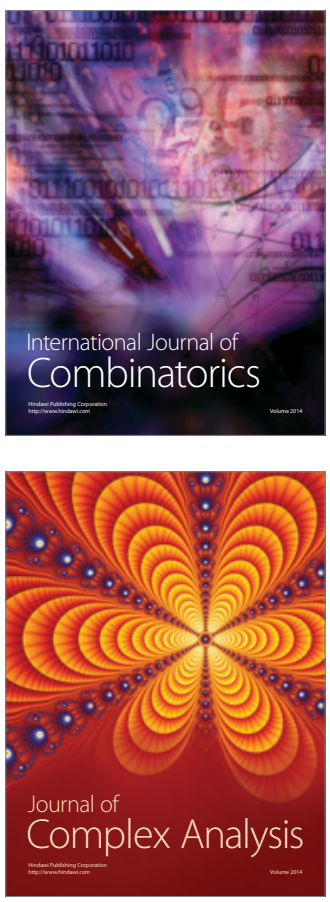

International Journal of

Mathematics and

Mathematical

Sciences
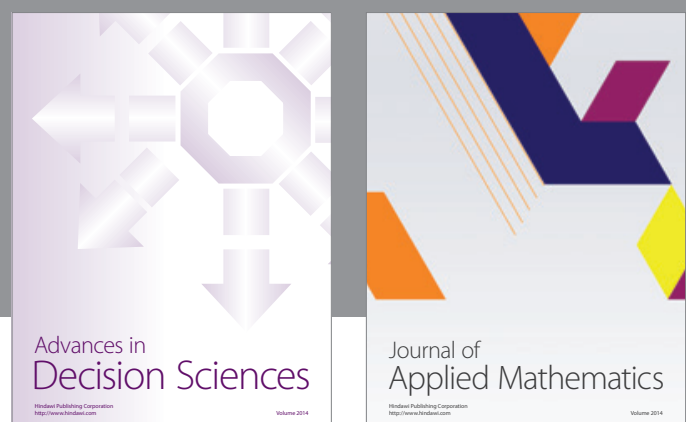

Journal of

Applied Mathematics
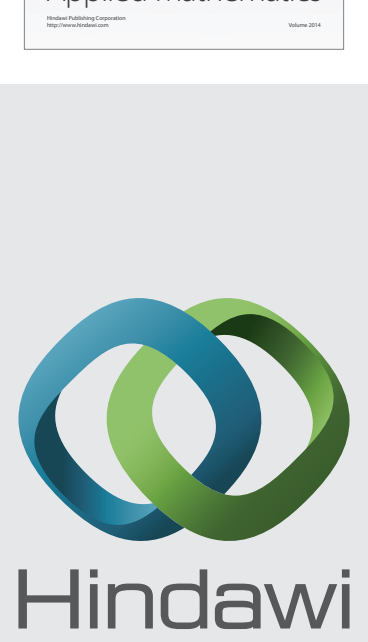

Submit your manuscripts at http://www.hindawi.com
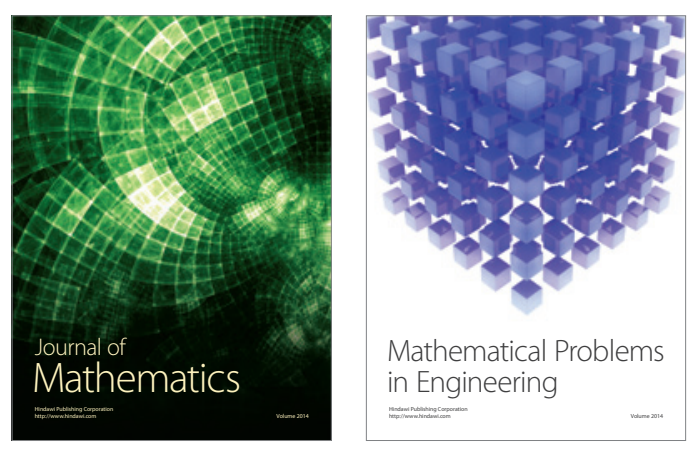

Mathematical Problems in Engineering
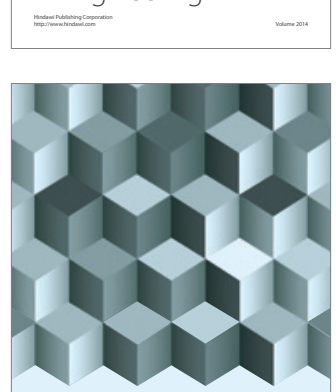

Journal of

Function Spaces
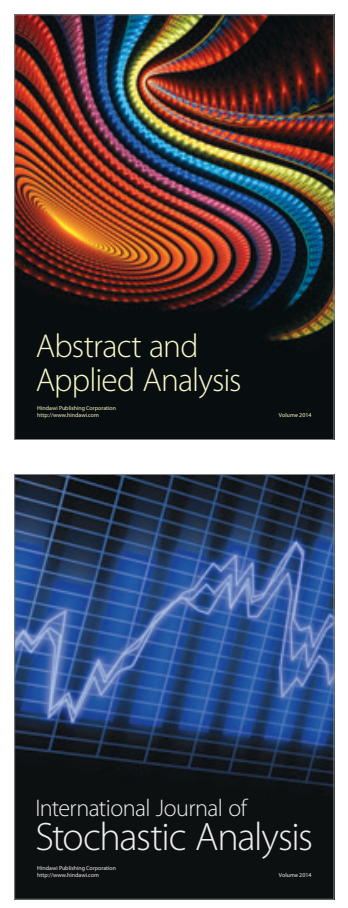

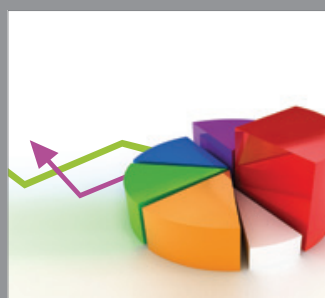

ournal of

Probability and Statistics

Promensencen
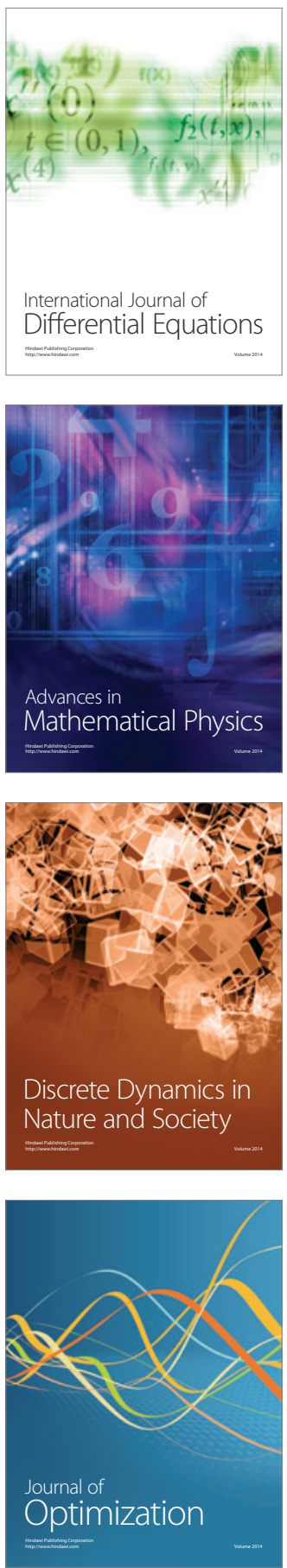